\title{
Dross formation and process parameters analysis of fiber laser cutting of stainless steel thin sheets
}

\author{
D. Teixidor ${ }^{(1)}$, J. Ciurana ${ }^{(1)}$, C. Rodríguez ${ }^{(2)}$ \\ (1) Department of Mechanical Engineering and Industrial Construction, Universitat de Girona, Girona, Spain. \\ daniel.teixidor@udg.edu \\ (2) Center for Innovation in Design and Technology, Tecnológico de Monterrey, Monterrey, Mexico.
}

\begin{abstract}
The coronary stent fabrication requires high precision profile cut. Fiber lasers present a solution to accomplish these requirements. This paper presents an experimental study of fiber laser cutting of $316 \mathrm{~L}$ stainless steel thin sheets. The effect of peak pulse power, pulse frequency and cutting speed on the cutting quality for fixed gas type and gas pressure were investigated. A mathematical model for the dross dimensions was formulated. The dross height and the dross diameter were analyzed and compared with the experimental results. This allows selecting the process parameters to reduce the dimensions of the dross deposited at the bottom of the workpiece.
\end{abstract}

Keywords: Laser cutting; fiber laser; cardiovascular stent, dross formation. 


\section{Introduction}

The laser cutting process has developed significantly over the past few decades and has become routine in sheet metal fabrication as a result of the attractive cutting velocities, excellent cut quality, processing flexibility as well as the widespread application possibilities that it affords [1].

One of its growing applications is the manufacturing of coronary stents for medical application. A stent is a wire mesh tube, which is deployed in a diseased coronary artery to provide smooth blood circulation. Stents can be either balloon expandable or self-expanding (using shape memory alloys). Stents are typically made from biocompatible materials such as stainless steel, nitinol (Ni-Ti alloy), cobalt-chromium, titanium, tantalum alloys, platinum iridium alloy as well as polymer. The most commonly used is stainless steel. The laser key requirement for its fabrication is a small consistent kerf width and this demands constant beam quality and excellent laser power stability. The laser cut must have a good surface quality with a minimum amount of slag and burr to reduce post-processing similarly the heat affected zone (HAZ) and molten material recast needs to be small [2].

Fiber laser is seen as an efficient, reliable and compact solution for micro machining which heat affected zone, kerf width and dross could be diminished to a minimum. This is because its important advantages as the combination of high beam power with high beam quality, small spot sizes, higher efficiency and almost free maintenance.

There are several research works, which use a fiber laser to study how the process parameters of the laser cutting affect the quality of the resultant surfaces. Kleine et al. [2] presented micro-cutting results in stainless steel samples of 100 and $150 \mu \mathrm{m}$ where the kerf width and the surface quality were analyzed. They studied also the laser conditions to minimize HAZ. They conclude that the fiber laser is capable to achieve very small diameters and small kerf widths presenting very similar features to those produced with a Nd:YAG laser. Muhammad et al. [3] investigate the basic characteristics of fiber laser cutting of stainless steel $316 \mathrm{~L}$ tube and understand the effect of introducing water flow in the tubes on minimizing back wall damages and thermal effect. The influence of laser parameters upon cutting quality for fixed gas type and gas pressure was investigated. Wet cutting enabled significant improvement in cutting quality. It resulted in narrower kerf width, lower surface roughness, less dross, absence of back wall damages and smaller HAZ. Laser average power and pulse width play a significant role in controlling the cutting quality. Increasing the pulse width increased beam/material interaction time, which increased the kerf width and surface roughness. Meng et al. [4] designed a cardiovascular stent cutting system based on fiber laser where the kerf width size was studied for different cutting parameters including laser output power, pulse length, repeat frequency, cutting speed and assisting gas pressure. Baumeister et al. [5] presented laser micro-cutting results for stainless steel foils with the aid of a $100 \mathrm{~W}$ fiber laser. Different material thicknesses were evaluated (100um to 300um). Processing was carried out with $\mathrm{cw}$ operation, and with nitrogen and oxygen assisting gases. Besides the high processing rate of oxygen assisted 
cutting, a better cutting performance in terms of a lower kerf width was obtained. Minimal kerf width of less $20 \mu \mathrm{m}$ was obtained with oxygen as the assisting gas. The kerf widths with nitrogen assisted gas were generally wider. Scintilla et al. [6] presented results of Ytterbium fiber laser cutting of Ti6Al4V sheets ( $1 \mathrm{~mm}$ thick) performed with Argon as cutting assistance gas. The effect of cutting speed and shear gas pressure on the HAZ thickness, squareness, roughness and dross attachment was investigated. The results show that, with increasing the cutting speed and then decreasing the heat input from at $2 \mathrm{~kW}$, an increase of HAZ and RL thickness occurs, up to $117 \mu \mathrm{m}$. Powell et al. [7] developed an experimental and theoretical investigation into the phenomenon of 'striation free cutting', which is a feature of fiber laser/oxygen cutting of thin section mild steel. The paper concludes that the creation of very low roughness edges is related to an optimization of the cut front geometry when the cut front is inclined at angles close to the Brewster angle for the laser- material combination. Yan et al. [8] carried out both experimental and 3D FE modeling studies to analyze the effects of process parameters on temperature fields, thermal-stress distribution and potential crack formation in high power fiber laser cutting of alumina. Based on the numerical and experimental results, the mechanism of crack formation in laser fusion cutting was revealed and crack- free cutting of thick-section alumina was demonstrated.

Other researchers studied the effect of process parameters on the fabrication of stents using different lasers on several materials like nitinol or stainless steel. Kathuria et al. [9] described the precision fabrication of metallic stent from stainless steel (SS 316L) by using short pulse $\mathrm{Nd}$ :YAG laser. They conclude that the processing of stent with desired taper and quality shall still be preferred by the short pulse and higher pulse repetition rate of the laser, which is desired to reduce further the heat affected zone as well as the wave depth of the cut section. Pfeifer et al. [10] Pulsed Nd:YAG laser cutting of $1 \mathrm{~mm}$ thick NiTi shape memory alloys for medical applications (SMA-implants). They studied the influence of pulse energy, pulse width, and spot overlap on the cut geometry, roughness and HAZ. They generated small kerf width ( $k$ $=150-300 \mu \mathrm{m})$ in connection with a small angle of taper $\left(\theta<2^{\circ}\right)$. Compared to short- and ultrashort-laser processing of SMA, high cutting speeds $(v=2-12 \mathrm{~mm} / \mathrm{s})$ along with a sufficient cut quality $(\mathrm{Rz}=10-30 \mu \mathrm{m})$ were achieved. The drawbacks can be seen in the higher thermal impact of the laser-material processing on the SMA, resulting in a HAZ (dimension: 6-30 $\mu \mathrm{m}$ ) which affects the material properties and the reduced accuracy of the cutting process. Shanjin et al. [11] presented Nd:YAG pulsed laser cutting of titanium alloy sheet to investigate the influences of different laser cutting parameters on the surface quality factors such as HAZ, surface morphology and corrosion resistance. The results presented show that medium pulse energy, high pulse rate, high cutting speed and argon gas at high pressure help to acquire thin HAZ layers. Also in comparison with air- and nitrogen-assisted laser cutting, argon-assisted laser cutting comes with unaffected surface quality. Yung et al. [12] performed a qualitative theoretical analysis and experimental investigations of the process parameters on the kerf profile and cutting quality. They micro-cut thin NiTi sheets with a thickness of $350 \mu \mathrm{m}$ using a $355 \mathrm{~nm} \mathrm{Nd:YAG} \mathrm{laser.} \mathrm{The} \mathrm{results} \mathrm{showed} \mathrm{that} \mathrm{the} \mathrm{kerf} \mathrm{profile} \mathrm{and} \mathrm{cutting} \mathrm{quality} \mathrm{are}$ significantly influenced by the process parameters, such as the single pulse energy, scan speed, 
frequency, pass number and beam offset, with the single pulse energy and pass number having the most significant effects. They obtained debris-free kerf with narrow width ( $\approx 25 \mu \mathrm{m})$ and small taper $(\approx 10)$. And concluded that as the single pulse energy is increased and the laser beam velocity is decreased, the kerf width increases. Muhammad et al. [13] studied the capability of picoseconds laser micromachining of nitinol and platinum-iridium alloy in improving the cut quality. Process parameters used in the cutting process have achieved drossfree cut and minimum extent of heat-affected zone (HAZ). Li et al. [14] reported investigations of femtosecond laser processing of NiTi SMA using a fundamental wavelength of $775 \mathrm{~nm}$ from Ti:Saphire laser and its second and third harmonic irradiations. They developed a thermal influence free optimal process to fabricate complex miniature SMA components. Huang et al. [15] studied the effect of a femtosecond laser machining on the surface and characteristics of Nitinol. The results have produced surface roughness of about $0.2 \mu \mathrm{m}$ on Nitinol. SEM and microstructural analyses revealed a HAZ smaller than $70 \mu \mathrm{m}$ in depth and a re-deposited layer of about $7 \mu \mathrm{m}$ exists on the machined surface. Raval et al. [16] machined a coronary stent after $\mathrm{Nd}$ :YAG laser cutting of 316LVM tubing and an assessment of its surface characteristics after electrochemical polishing. Finally, Scintilla et al. [17] analyzed the influence of processing parameters and laser source type on cutting edge quality of AZ31 magnesium alloy sheets and differences in cutting efficiency between fiber and $\mathrm{CO} 2$ lasers were studied. They investigated the effect of processing parameters in a laser cutting of $1 \mathrm{~mm}$ and $3.3 \mathrm{~mm}$ thick sheets on the cutting quality. Their results showed that productivity, process efficiency and cutting edges quality obtained using fiber lasers outperform $\mathrm{CO} 2$ laser performances.

Some authors studied the formation of the dross developing analytical models in order to predict the shape of this melt material attached to the cutting edge. Yilbas et al. [18] formulated a mathematical model to predict the melt thickness and the droplet diameter. They compared it with experimental results of a $\mathrm{CO} 2$ laser obtaining good fit. They found that the liquid layer thickness increases with increasing laser output power and reduces with increasing assisting gas velocity. Tani et al. [19] evaluated the 3D geometry of the cutting front of the melting film considering mass, force and energy balance in an analytical model. Schuöcker et al. [20] presented a model for the dynamic behavior of the liquid layer in laser cutting that predicts the melt ejection. They related the droplet ejection and the formation of periodic striations along the cut edges. Shuja et al. [21] simulated the temperature field and the phase change in the heated region. They examined the influence of laser power intensity and scanning speed on temperature field and melt depth.

The present work aims to investigate the characteristics of fiber laser cutting of stainless steel 316L-based cylindrical stents. The effect of laser cutting parameters on the cutting quality for fixed gas type and gas pressure was investigated. Therefore, machining stent geometries in stainless steel sheets, this work will contribute to understand the relation between the process parameters and the responses studied. The melt depth and dross size are mathematical modeled. The dross experimental values are compared with the predictions. 


\section{Methodology or Experimental Procedure}

The laser source employed in this work was a Rofin FL x50 Fiber Laser. This is a multi-mode laser capable of delivering up to $500 \mathrm{~W}$ power at $1080 \mathrm{~nm}$ wavelength and a beam quality factor, $M<1.1$. The output can be modulated with a pulse frequency up to $5 \mathrm{kHz}$. The shortest pulse duration is $26 \mu \mathrm{s}$. The process fiber used was $150 \mu \mathrm{m}$ in diameter, which was mounted in a focusing optics consisted of a Precitec Fine Cutting head with a collimation lens of $50 \mathrm{~mm}$ length and a $50 \mathrm{~mm}$ focal length objective. The focused spot size was calculated to be $150 \mu \mathrm{m}$. The coaxial assist gas nozzle had an exit diameter of $0.5 \mathrm{~mm}$. The system is integrated in a Kondia CNC machine, which controls the movement of $X, Y$ and $Z$ stages for translating the work under the focused laser spot.

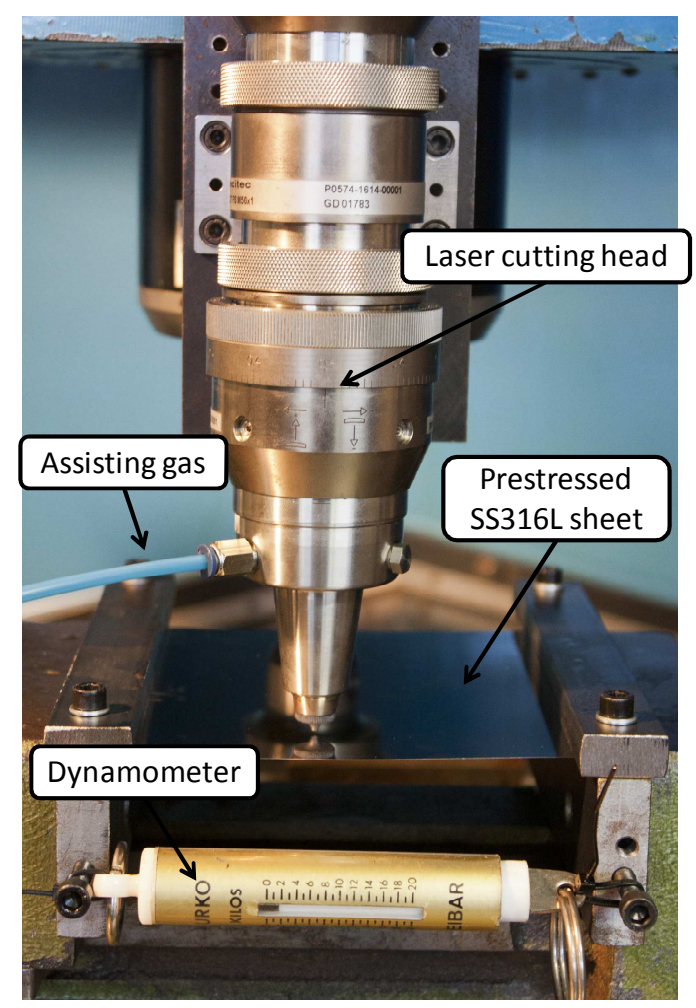

Figure 1 Set-up of the laser system.

In this work Stainless Steel $316 \mathrm{~L}$ sheets of $100 \mu \mathrm{m}$ thickness were used as a workpiece material. These sheets were clamped and prestressed, with an approximately $2 \mathrm{~N}$ tension, in order to avoid its deformation due the temperature. In this way the standoff distance is kept constant. Figure 1 presents the set-up of the system with the prestressed sheets and the laser cutting head in working position. 


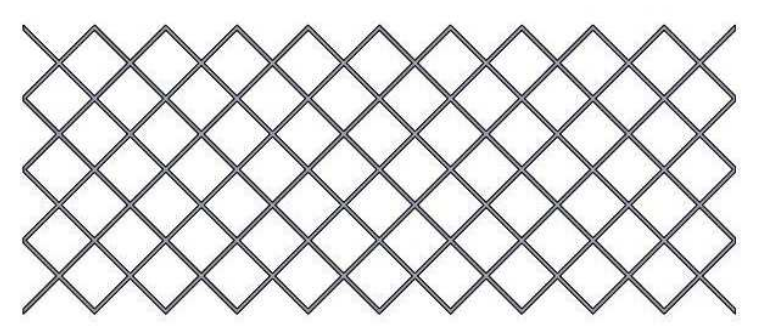

Figure 2 Stent geometry used on the laser cutting experiments.

Preliminary screening experiments were carried out to determine the appropriate processing parameters levels to be used for the design of experiments. A full factorial design was used to determine the effects of peak pulse power, cutting speed and pulse frequency on the resultant cutting quality. The factors and factor levels utilized in this work are summarized in Table 1Table 1.. These factor levels results in a total of 27 unique factor level combinations. Nitrogen was used as the assist gas to protect the optics and to remove the molten material from the cut kerf. The pressure was 6 bar, the stand-off distance between the nozzle tip and the workpiece surface was $0.2 \mathrm{~mm}$ and the pulse duration was $125 \mu \mathrm{s}$. These parameters were studied in the preliminary experiments and decided to keep constant for all the experiments. In order to investigate the effects of the process parameters on the cutting quality factors, stent simple geometry [9], as is presented in Figure 2, was used as a cutting shape for the experiments. The samples have dimensions of $20 \times 8.5 \mathrm{~mm}$ with strut width of $0.1 \mathrm{~mm}$. With this flat geometry a cylindrical structure could be formed by rolling and adhesive joining [22], but it is not the aim of this work. The cutting quality factors investigated were kerf width, surface roughness, dross deposition, and heat effects.

Table 1. Factors and factor levels of the design of experiments.

\begin{tabular}{lcccc}
\hline Peak pulse power & W & 200 & 300 & 400 \\
Pulse frequency & $\mathrm{Hz}$ & 3000 & 4000 & 5000 \\
Cutting Speed & $\mathrm{mm} / \mathrm{min}$ & 250 & 375 & 500 \\
\hline
\end{tabular}

Characterization of the laser cut samples was conducted by confocal microscope Axio CSM 700 from Carl Zeiss. The kerf width is measured and the surface roughness is calculated by the microscope in conformity with the DIN EN ISO 4287 standard. The surfaces are also analyzed in 3D images from the top and the cut edges. The measurements of the dross area were performed with an optical microscope LEICA DMR-XA attached to NIKON F90 and RICOH XRX3000 camera bodies and digital video with a SONY DXC950-P of 3CCD camera for the collection of digital images. These images were numerically processed using the Quartz PCIC software, version 5 . 


\section{Results and Discussion}

A total number of 27 stents were machined with laser cutting process by following the experimental plan discussed in previous section. Kerf width, surface roughness and dross deposition were measured as a quality factors. The surface roughness of four of the samples was not measured, and the dross deposition was just measured in 20 of the geometries machined. Some statistical analysis will be presented in order to identify the relations between the process parameters and the responses.

\subsection{Kerf width}

Figure 3 shows the influences of cutting parameters upon the kerf width. It presents how each process parameter affects the kerf width. The results clearly show that the kerf width increased as the peak pulse power, pulse frequency and cutting speed increased. As expected the increase of peak pulse power and pulse frequency results in bigger kerf width. During the cutting process, the average power is proportional to these parameters and the pulse width. Thus, higher average power results in bigger kerf width. On the other hand would be expected that the increasing of the cutting speed will lead to a reduction of the kerf width [3, 4]. As the cutting speed reduces, the interaction time between laser beam and material increases which creates a larger kerf. However, the results present the opposite trend. This happens because the range used ( 250 to $500 \mathrm{~mm} / \mathrm{min}$ ) is not big enough to see the real trend of the relation between cutting speed and kerf width. Muhammad et al. [3] pointed out that the relation between both parameters increased until $1000 \mathrm{~mm} / \mathrm{min}$ where the kerf width started to decrease after this point. The kerf widths obtained in the experiments were within the range of 150 to $230 \mu \mathrm{m}$. Minimum kerf was obtained at lower peak pulse power, $200 \mathrm{~W}$, lower pulse frequency, $3000 \mathrm{~Hz}$ and lower cutting speed, $250 \mathrm{~mm} / \mathrm{min}$. Although, the minimum achieved corresponds to the theoretical spot size calculated, it is clear that the experimental values obtained are bigger than this theoretic one. This was expected, Muhammad et al. [3] presented kerf width values $60 \%$ above the theoretic spot size when the pulse peak power or the pulse frequency is increased and Baumester et al. [5] showed that increasing the energy the kerf width become $200 \%$ higher than the focal diameter. 


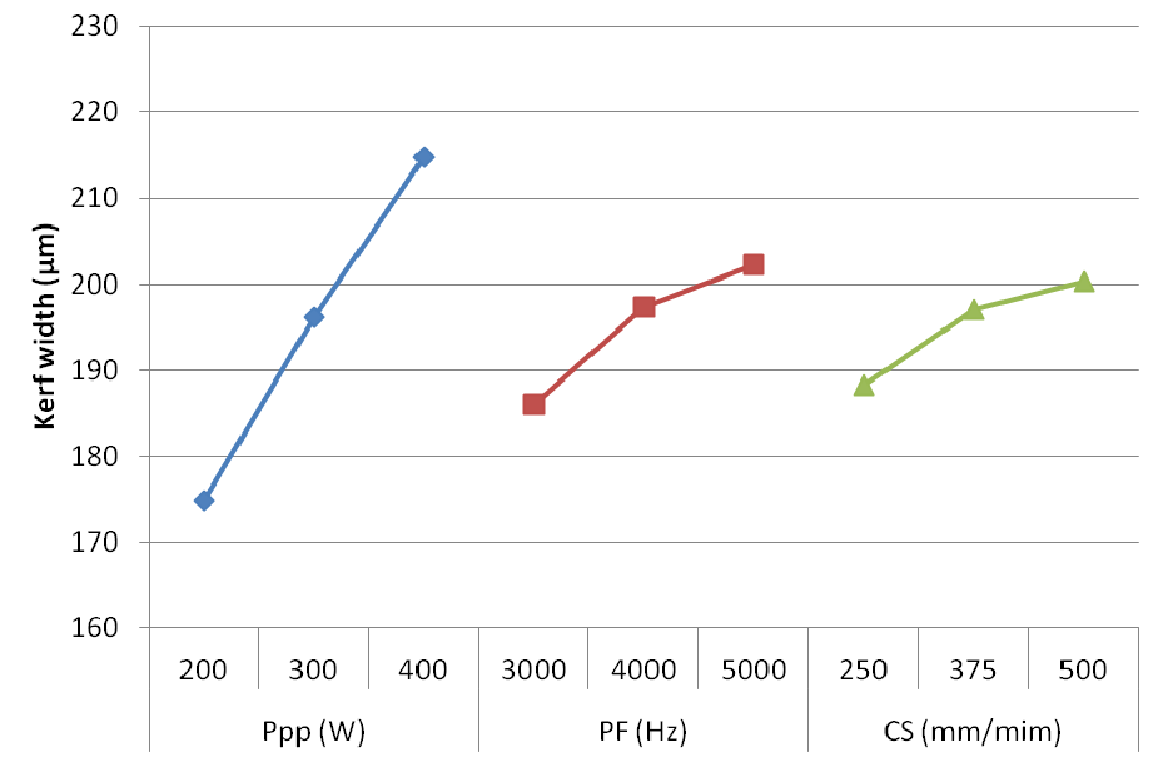

Figure 3. Kerf width as a function of laser cutting parameters: peak pulse power, pulse frequency, and cutting speed.

\subsection{Surface roughness and Striation on the cut surface}

Analysis was carried out to characterise the topography of the cut edges to determine the surface finish quality. The average roughness was measured in the mid section of the cut edge surface. Three measurements were taken in three different struts of each stent. The mean of these measures has been used as the experimental results for the surface roughness analysis. Figures 4 and 5 show the image of the cutting edge and the surface roughness profile obtained by the confocal microscope for two of the samples.
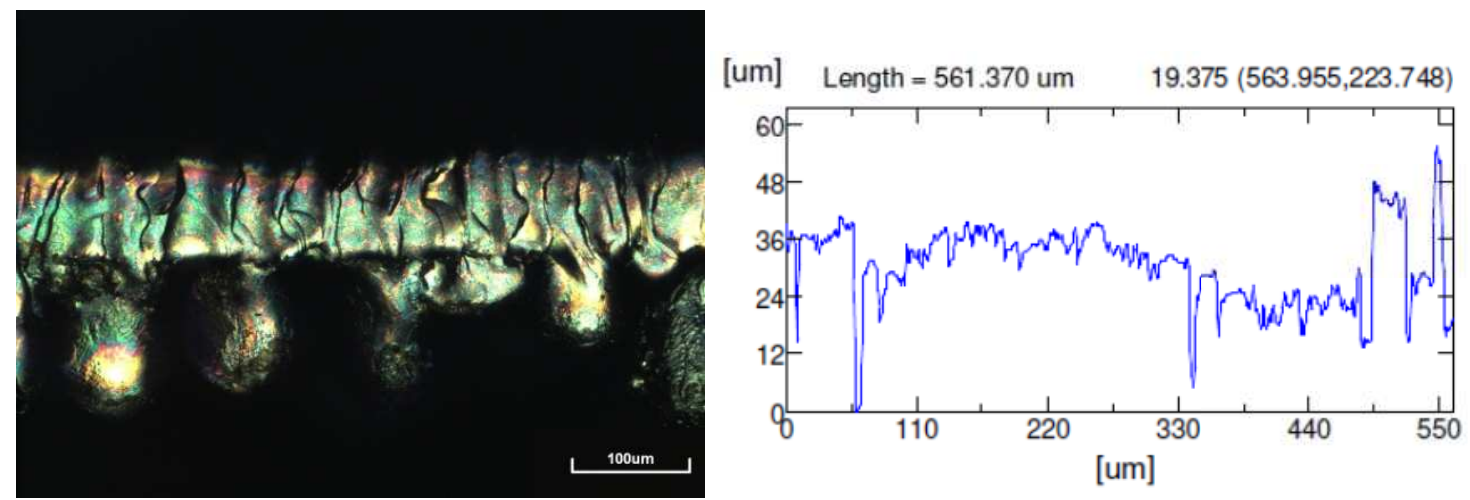

Figure 4. Surface roughness of the cut surface for pulse power $400 \mathrm{~W}$, pulse frequency $3000 \mathrm{~Hz}$ and cutting speed $500 \mathrm{~mm} / \mathrm{min}$. a) Image of the cut surface from confocal microscope (219X). b) surface roughness profile across the cut edge. Where $\mathrm{Ra}=1,447$ 

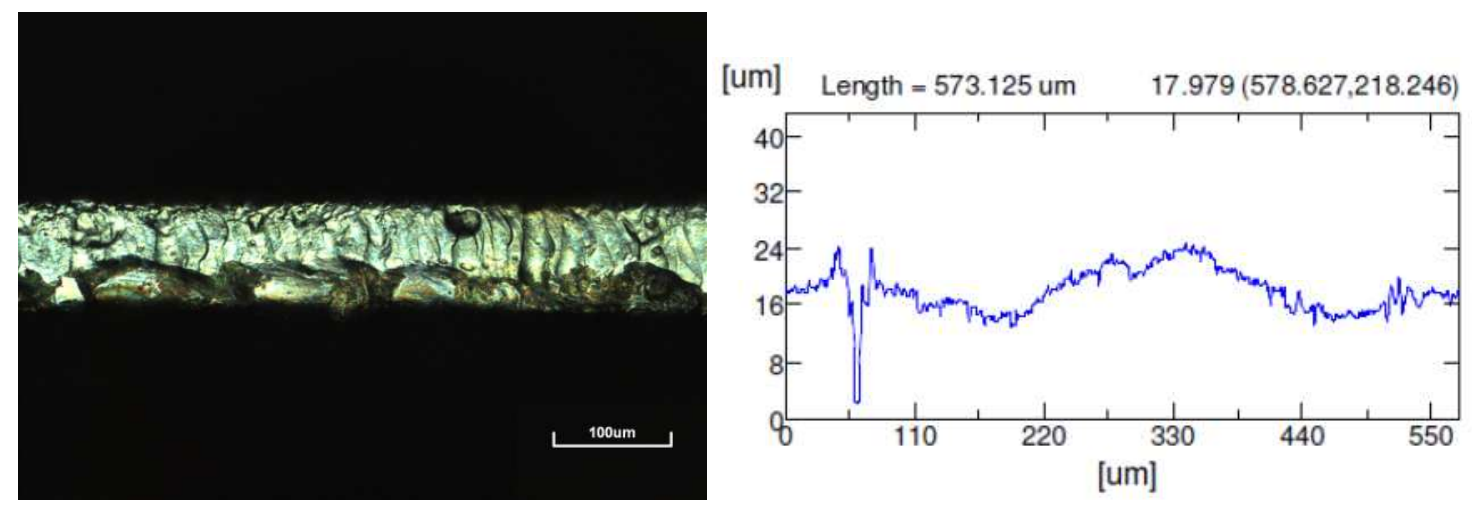

Figure 5. Surface roughness of the cut surface for peak pulse power $300 \mathrm{~W}$, pulse frequency $3000 \mathrm{~Hz}$ and cutting speed $375 \mathrm{~mm} / \mathrm{min}$. a) Image of the cut surface from confocal microscope (219X), b) surface roughness profile across the cut edge. Where $\mathrm{Ra}=0668$,

Figure 6 shows the relationship between the surface roughness and the laser parameters. Thus the influence of the process parameters upon the surface roughness is presented. The surface roughness increased with the increasing peak pulse power. Although between the two levels seems to keep constant, clearly for the higher peak pulse power the surface roughness presents its higher values. Although, higher pulse frequency leads to higher average power, it results in better surface roughness due to high pulse overlapping. Lower cutting speed also increases the overlapping, thus higher cutting speed results in worst surface roughness as the results pointed out. The surface roughness obtained in the experiments was within the range of 0,547 to $1,447 \mu \mathrm{m}$. Although there are some parameters combinations with higher values of surface roughness, this range is similar to the ones presented in other works [2, 3]. Minimum Ra was obtained at medium peak pulse power, $300 \mathrm{~W}$, medium pulse frequency, $4000 \mathrm{~Hz}$ and lower cutting speed, $250 \mathrm{~mm} / \mathrm{min}$.

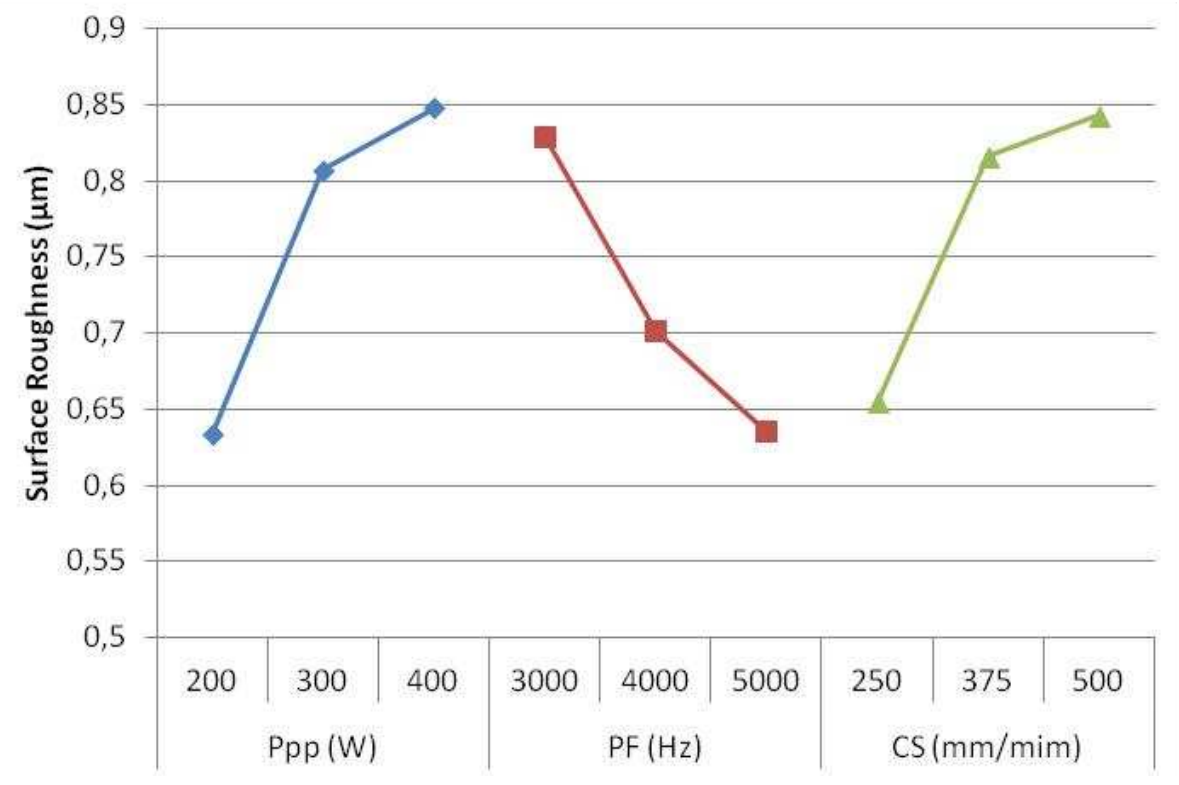

Figure 6. Surface roughness as a function of laser cutting parameters: peak pulse power, pulse frequency, and cutting speed. 
The striation refers to periodic lines appeared on the cut surface. These periodic lines reflect the effect of the combination of the laser beam moving along the surface the pulse frequency and the pulse duration, generating pressure gradients across the cut kerf and varying vaporisation fronts. This phenomenon created regularly spaced striations and increased the surface roughness on the cut surface. Thus, striation is directly related to the surface roughness and mainly affects the surface quality of the cut zones. The profiles of the figures 4 and 5 indicate that striation occur, presenting different height depending on the parameters. However, looking at the images it is not easy to see a clear pattern related to the striations. It seems that the molten material is deposited on the cut surface modifying these striations generated from the laser beam movement.

\subsection{Heat effects}

Heat effect on the surrounding material is a critical factor in cutting thin materials especially in medical device application. Small and thin materials are very sensitive to thermal distortion. Experiment results show that low average power reduced the thermal distortion. Thus, when pulse peak power and pulse frequency are higher it resulted in a noticeable thermal effect and surface oxidation along the cut as clearly shows the figure 7 .
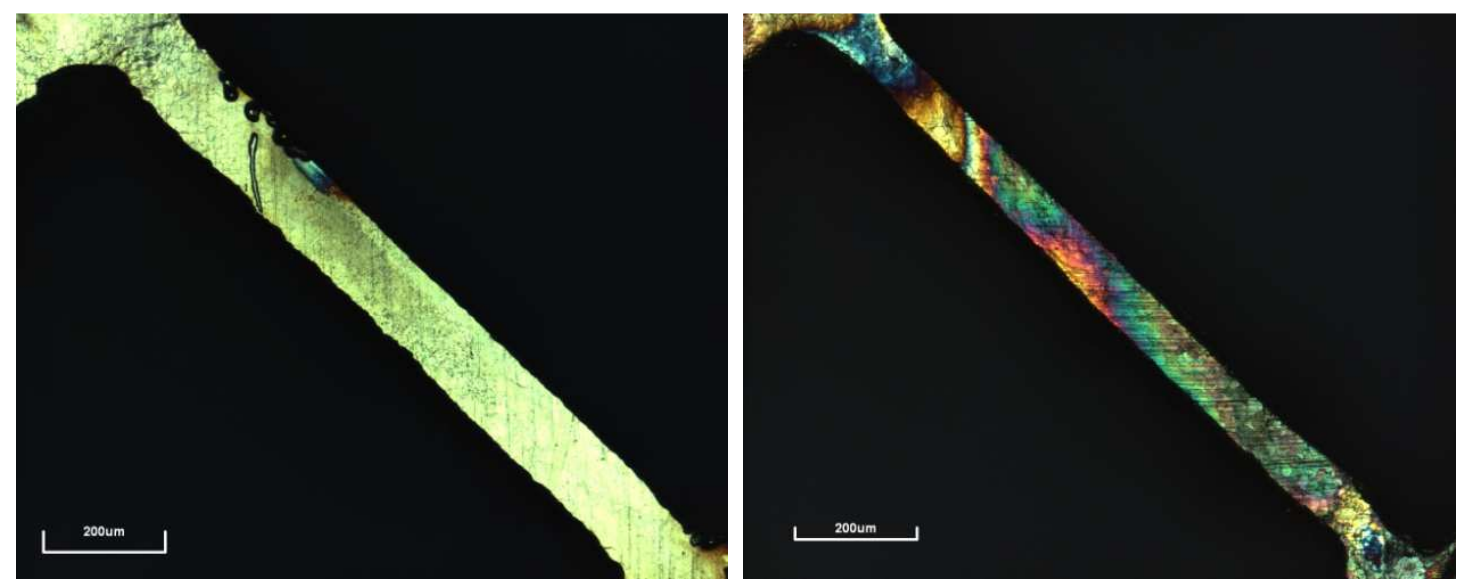

Figure 7 Comparison of the thermal effect along the strut; a) pulse peak power 200, pulse frequency $3000 \mathrm{~Hz}$ and cutting speed $250 \mathrm{~mm} / \mathrm{min}$, b) peak pulse power 400, pulse frequency $5000 \mathrm{~Hz}$ and cutting speed $500 \mathrm{~mm} / \mathrm{min}$.

\subsection{Dross deposition}

The images of the cut surface with the dross formed where obtained from destructive tests in which the specimens were embedded in resin. Figure 8 and 9 present images from the cut surface and from the bottom of the geometry with the dross deposited for two different samples. Clearly there is much dross deposited in the cut edges of the samples. Depending on the combination of the process parameters there is different amount of it. The area was measured from the cut surface images in order to establish a relation between the input parameters and the dross formation. 

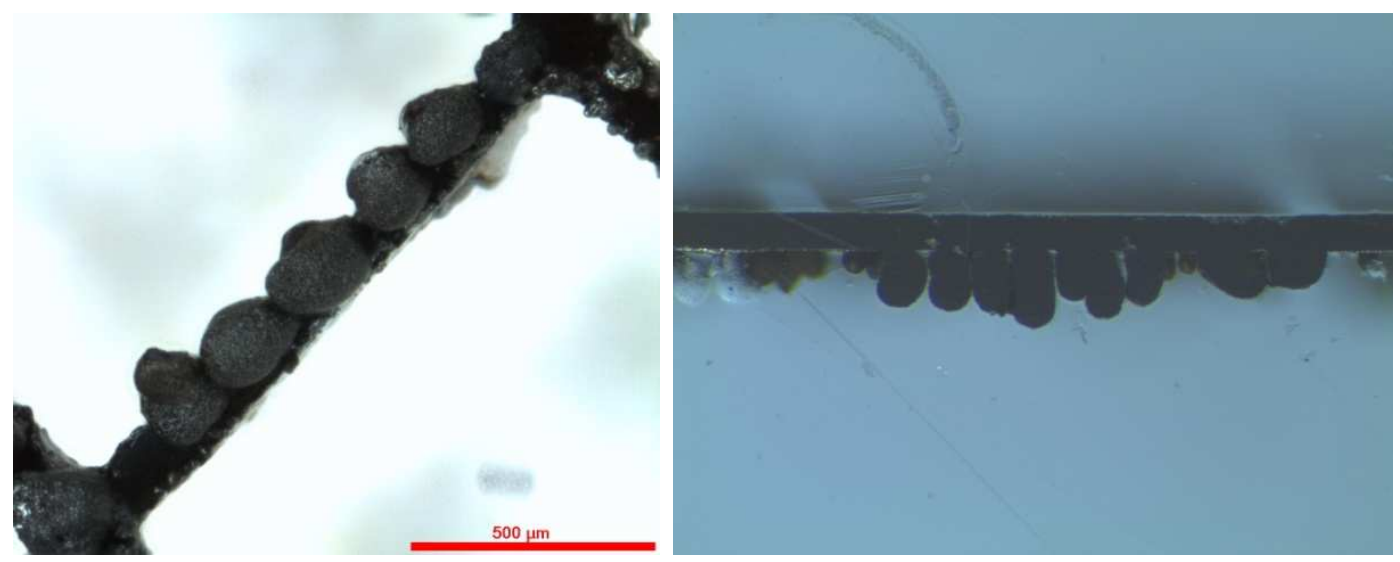

Figure 8. Dross deposition for peak pulse power $300 \mathrm{~W}$, pulse frequency $3000 \mathrm{~Hz}$ and cutting speed $250 \mathrm{~mm} / \mathrm{min}$ sample a) bottom image from optical microscope (50X), b) cut surface image from optical microscope (80X).
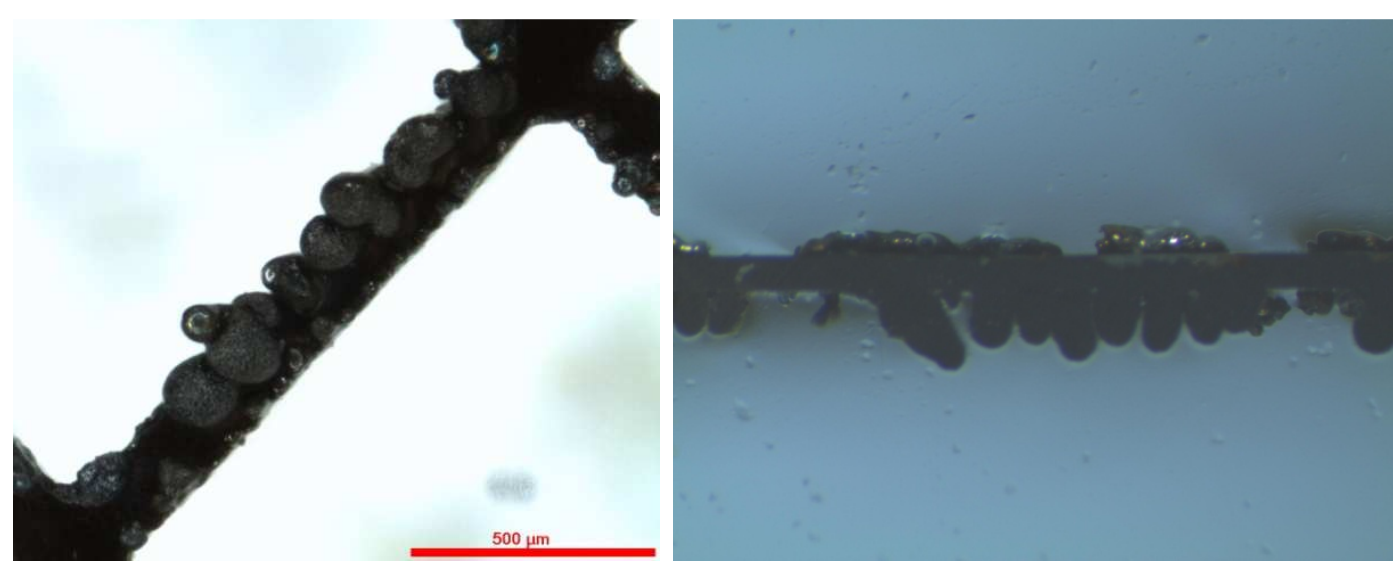

Figure 9. dross deposition for peak pulse power $300 \mathrm{~W}$, pulse frequency $4000 \mathrm{~Hz}$ and cutting speed $500 \mathrm{~mm} / \mathrm{min}$ sample a) bottom image from optical microscope (50X), b) cut surface image from optical microscope (80X).

The dross deposition has been measured for each stent in six different struts. The area of the dross has been taken from the upper part and lower part of the stent separately. The mean of the six measurements has been used as the value for the results analysis.

Figure 10 presents the dross deposition area as function of the laser cutting parameters. Clearly the samples processed were not free from dross. The molten material was not totally ejected out from the cut kerf and attached to the bottom side of the cut wall. The dross deposition area increased as the peak pulse power, pulse frequency and cutting speed increased. The relation presented is similar at the one for the kerf width. If the peak pulse power and the pulse frequency increase the average power increases leading to more molten material. More molten material means that is more difficult to remove by the assist gas. At the same time higher cutting speeds reduce the time the gas is working on the same area, thus the dross area gets bigger. 


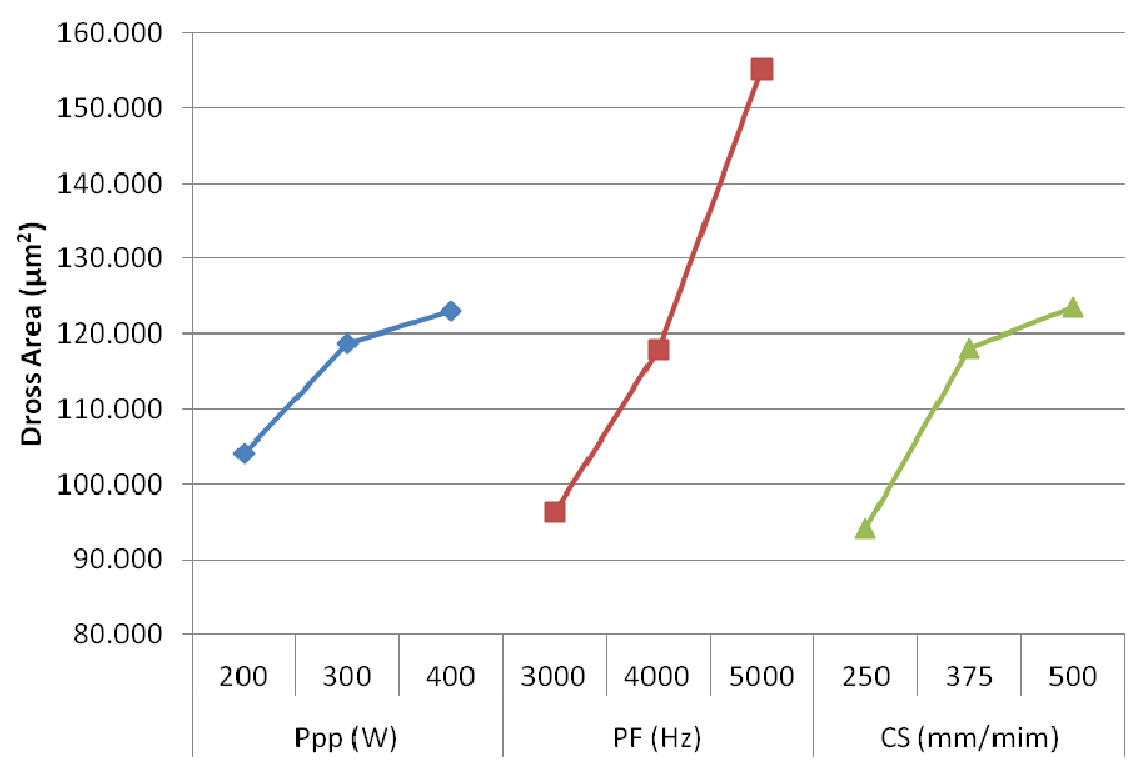

Figure 10. Dross deposition area as a function of laser cutting parameters: peak pulse power, pulse frequency, and cutting speed.

As can be seen in the figure $9 \mathrm{~b}$, there are some cases where the sample presents material formation on the top of the struts. This material rejected from the cutting zone, has been deposited on the sheet in droplets form. Thus, although these droplets are not always on the cut edge, the area of this material formed has been also measured following the same procedure described before.

Table 2. Workpiece and assisting gas properties used in the simulations.

\begin{tabular}{lll}
\hline Property & Value & Units \\
\hline Boiling temperature & 3133 & $\mathrm{~K}$ \\
Melting temperature & 1648 & $\mathrm{~K}$ \\
Density of assisting gas & 6.875 at $6 \mathrm{bar}$ & $\mathrm{kgm}^{-3}$ \\
Density of workpiece & 7990 & $\mathrm{kgm}^{-3}$ \\
Specific heat capacity & 500 & $\mathrm{Jkg}^{-1} \mathrm{~K}^{-1}$ \\
Latent heat of melting & $2.75 \times 10^{5}$ & $\mathrm{Jkg}^{-1}$ \\
Viscosity of melting material & $0.9 \times 10^{-2}$ & $\mathrm{Nsm}^{-2}$ \\
Viscosity of assisting gas & $61.77 \times 10^{-6}$ & $\mathrm{Nsm}^{-2}$ \\
Velocity of gas jet & 417 & $\mathrm{~ms}^{-1}$ \\
\hline
\end{tabular}




\section{Mathematical modelling}

As shown in the results the dross deposition is quite important. Thus, a mathematic model is presented in order to predict the dimensions of this dross as function of some of the laser cutting parameters. In this way, we can select the proper laser conditions to reduce the amount of dross. The height and the diameter of the dross deposited on the experimental samples was measured and compared with the model predictions.
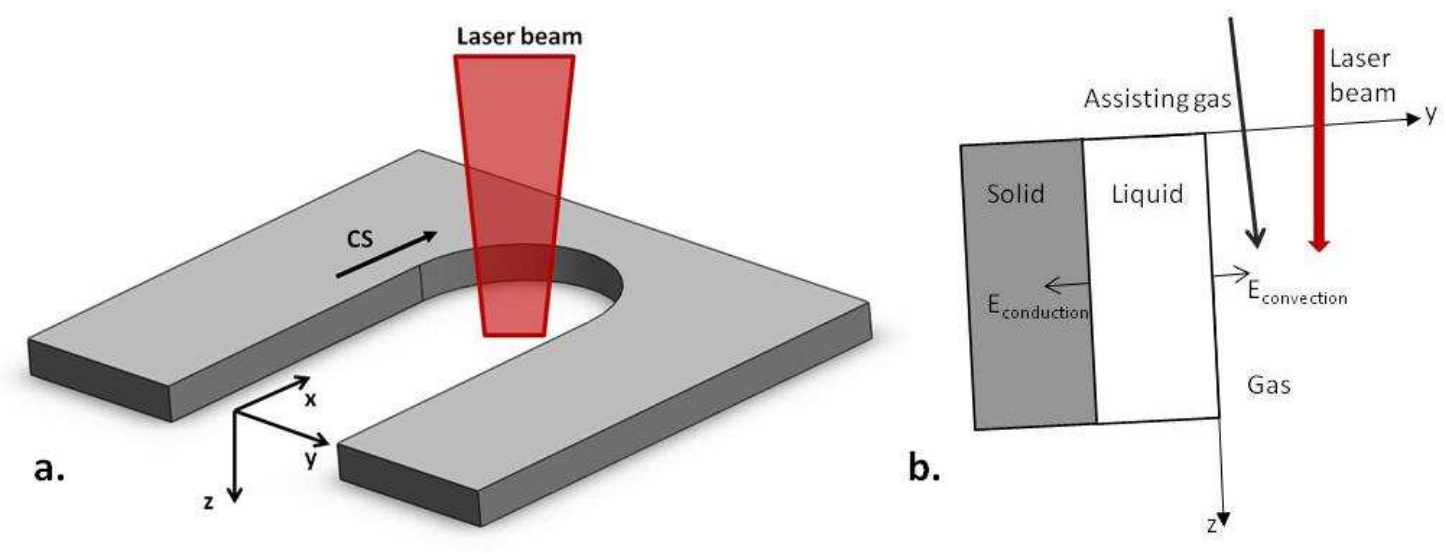

Figure 11. a) Schematic view of laser cutting process. b) Schematic view of melt section.

\subsection{Dross height}

Considering the laser cutting as a transient process where a Gaussian laser beam strikes the surface of a substrate, moving in the positive $x$ direction with a uniform cutting speed. The laser beam intensity can be described by the Gaussian distribution as follows:

$I(x, y, z)=\frac{P}{\pi R^{2}} e^{-\left[\left(x^{2}+y^{2}\right) / R^{2}\right]}$

The process is considered continuous wave operation. The high repetition rates and the levels of cutting speed used during the experiments result in high overlapping between pulses (98.15-99.44\%), calculated as presented in [23] making the assumption of the continuous wave acceptable. The convection and conduction are considered negligible.

Thus, the complex three dimensional kerf, as shown in Figure 11a, can be separated into finite surface elements described by $x$ and $y$ directions. For each element the energy balance can be described as follows; the laser input energy is equal to the energy necessary for the phase change of the surface.

$E_{\text {beam }} \delta x \delta y=E_{\text {melt }} \delta x \delta y$

The laser input energy is given by 
$E_{\text {beam }}=\int_{-\infty}^{\infty} I(x, y, z) \frac{\partial x}{C S}=\frac{a P}{C S \sqrt{\pi} R} e^{-\left(y^{2} / R^{2}\right)}$

Where $a$ is the absorptance of SS316L at the Nd:YAG laser wavelength $1.064 \mu \mathrm{m}$. The phase change energy is given by:

$E_{\text {melt }}=\rho\left[C_{p s}\left(T_{m}-T_{i}\right)+L_{m}+C_{p m}\left(T_{v}-T_{m}\right)\right] d_{H}(y) \partial x \partial y$

Where $d_{H}(y)$ is the dross height at $y$. Setting the energy balance yields

$\frac{a P}{C S \sqrt{\pi} R} e^{-\left(y^{2} / R^{2}\right)} \partial x \partial y=\rho\left[C_{p s}\left(T_{m}-T_{i}\right)+L_{m}+C_{p m}\left(T_{v}-T_{m}\right)\right] d_{H}(y) \partial x \partial y$

Then, the channel depth can be obtained as

$d_{H}(y)=\frac{a P}{C S \sqrt{\pi} R \rho\left[C_{p s}\left(T_{m}-T_{i}\right)+L_{m}+C_{p m}\left(T_{v}-T_{m}\right)\right]} e^{-\left(y^{2} / R^{2}\right)}$

\subsection{Liquid layer thickness}

Considering the melt layer generated at the solid surface during the steady laser heating process (Figure 11b). The influence of assisting gas on the cutting process needs to be modeled prior to dross diameter formulation. This is because the droplet diameter depends on the liquid layer. The rate of energy balance with the melting process is considered in order to determine the liquid layer thickness.

$\dot{E}_{\text {beam }}+\dot{E}_{\text {oxidation }}=\dot{E}_{\text {melt }}+\dot{E}_{\text {cond }}+\dot{E}_{\text {conv }}$

It is considered that the generated melt layer flows steadily in the direction of the assisting gas due to the drag force developed at the assisting gas-liquid interface. Because of the micrometric dimensions of the sheet thickness and the kerf width, it is assumed that the rate of energy convected ( $E_{\text {conv) }}$ from the surface to the assisting gas, and the rate of energy conducted $\left(E_{\text {cond }}\right)$ from the melted material to the solid substrate are minimal compared with the incident beam energy. Also the use of nitrogen as an assisting gas reduces to a minimum the exothermic reaction which would contributed to the energy transport process at the interface ( $\left.E_{\text {oxidation }}\right)$.

The energy of the melting or the phase change can be written as:

$\dot{E}_{m e l t}=\dot{m}_{L}\left[C_{p s}\left(T_{m}-T_{i}\right)+L_{m}+C_{p m}\left(T_{v}-T_{m}\right)\right]$

where $C_{p s}$ and $C_{p m}$ are the specific heat capacity of the material in the solid and the liquid state, respectively, $T_{i}, T_{m}$ and $T_{v}$ are the initial temperature, the melting temperature and the vaporization temperature of the material, respectively. $\dot{m}_{L}$ is the rate of mass generated from solid into liquid at the solid surface which in laser melting process, can be written as 
$\dot{m}_{L}=\frac{\partial}{\partial t}(\rho V)=\rho_{L} v_{L} A$,

Where $v_{L}$ is the velocity of the molten material, $\rho_{L}$ is the density of the molten metal, $A$ is the cross sectional area. Setting the rate of energy balance across the melt per unit area yields

$\frac{\dot{E}_{\text {beam }}}{A}=\frac{\dot{E}_{\text {melt }}}{A}$

Therefore,

$v_{L}=\frac{P}{A \rho_{L}\left[C_{p s}\left(T_{m}-T_{i}\right)+L_{m}+C_{p m}\left(T_{v}-T_{m}\right)\right]}$

Considering that the movement in the molten material towards the bottom of the workpiece is induced by a shear stress exerted by the assisting gas jet on the surface of the molten zone [20]. The melt's velocity is given by

$v_{L}=\frac{s_{L} \cdot \tau}{\mu_{L}}$

Where $s_{L}$ is the liquid layer thickness, $\mu \mathrm{L}$ is the dynamic viscosity of the molten metal, and $\zeta$ is the shear stress which is given by the following equation.

$\tau=\sqrt{\frac{\rho_{G} \mu_{G} v_{G}^{3}}{z}}$

Where $\rho_{G}$ is the density of the gas, $\mu_{G}$ is the dynamic viscosity of the gas, $v_{G}$ is the gas velocity, and $z$ is the sheet thickness. Introducing equations (6) and (7) into equation (4) yields

$S_{L}=\frac{\mu_{L} P}{A \rho_{L}\left[C_{p s}\left(T_{m}-T_{i}\right)+L_{m}+C_{p m}\left(T_{v}-T_{m}\right)\right]}\left(\frac{\rho_{G} \mu_{G} v_{G}^{3}}{z}\right)^{-1 / 2}$

\subsection{Dross diameter}

The dross formation depends on the liquid properties, such as viscosity, density and surface tension, as well as laser and cutting properties, such as assisting gas velocity, kerf size and liquid layer thickness. Moreover, a laminar flow of liquid film breaks up into droplets in an orderly and periodic manner. In practice small satellite droplets can also be formed in between the main droplets due to high aerodynamic forces and instabilities associated with the breakup process. The formulation of drop formation based on the ligament disintegration can be appropriate to a laser cutting process. Droplet diameter $\left(d_{D}\right)$ formulated earlier is adopted herein [18]:

$d_{D}=\left(\frac{3 \pi}{\sqrt{2}}\right) s_{L}\left(1+\frac{3 \mu_{L}}{\sqrt{\rho_{L} s_{L}}}\right)^{1 / 6}$ 
Equations (6) and (15) are used to compute the dross height and diameter in the cutting sections, respectively.

The dross height and diameter is analyzed and compared with the experimental results. Table 2 gives the material and assisting gas properties. The diameter and height of the dross presented as experimental results are obtained by taking the average diameter of the measures taken in 5 different struts of each sample. The diameter measures are taken from bottom images like figure $8 \mathrm{a}$ and the height measures are taken from cut edge front images like figure $8 \mathrm{~b}$.

Figure 11 presents the dross height predicted from equation 6 as a function of peak pulse power for different laser cutting speeds. As in the previous case, the height of the dross increases with the increasing of the pulse peak power. In this sense, the predicted height and the average measured show similar tendencies. The predicted lines show that the height of the dross reduces when the laser cutting speed increases and the experimental results present the opposite trend. In a laser milling process lower cutting speed results in higher material removal because the incident laser beam lasts longer in the same area. However, in the laser cutting process, the presence of the assisting gas changes the trend. As lower is the cutting speed more time is working the gas to eject the molten material, reducing the height dross. Although there is no much literature relating the dross dimension with the cutting speed, this is in the line with the results showed by Tani et al. [19]. Thus, the effect of the cutting speed in the model must be understood as the inverse of the reality. However further research is necessary to ensure this relation.

Figure 12 shows the dross diameter predicted from equation 15 with peak pulse power. Experimentally, obtained dross diameters are given for comparison. The higher pulse peak power results in bigger dross diameter. The Increasing of power intensity increases the liquid film thickness, which in turn enhances the dross diameter. On comparing the predictions with the average dross diameter measured both results agree quite well. However, both trend lines present different slopes. This may be associated to the assumptions made in the analysis. 


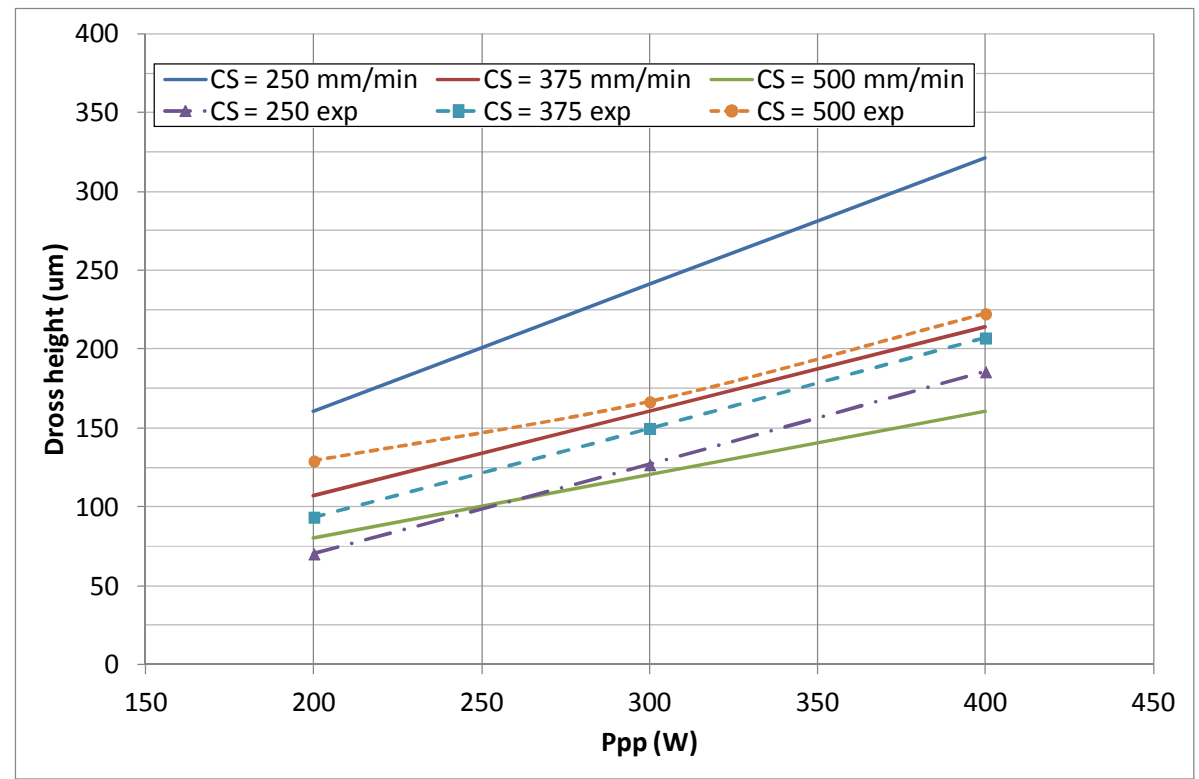

Figure 11. Predicted and experimental dross diameter as a function of peak pulse power for different laser cutting speeds.

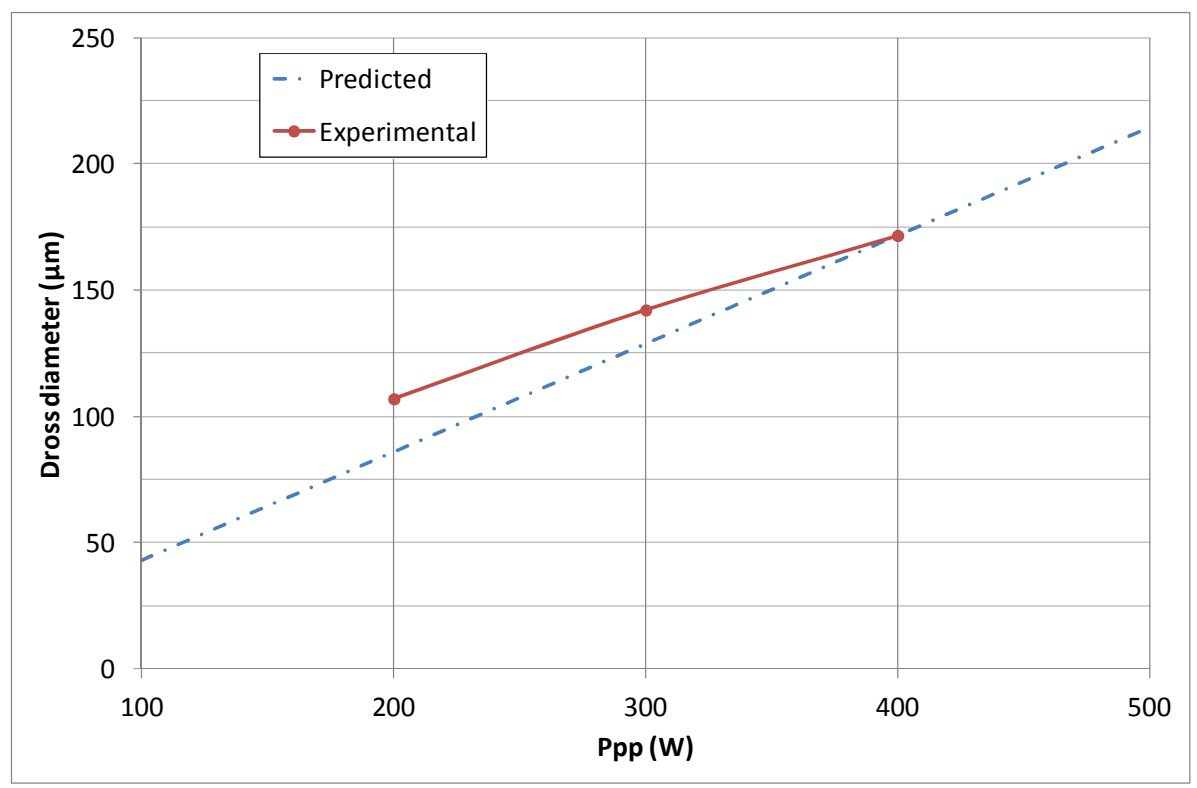

Figure 12. Predicted and experimental dross diameter as a function of peak pulse power.

\section{Conclusions}

In this study experimental results of fiber laser cutting of stainless steel 316L sheets were reported. The effect of peak pulse power, pulse frequency and cutting speed on the cutting quality for fixed gas type and gas pressure were investigated. The analysis showed that increasing the peak pulse power and the cutting speed increases the kerf width, surface roughness and dross deposition. However, the effect of the cutting speed needs further 
research because with higher values the dross and the kerf width are expected to decrease. Higher pulse frequency values result in bigger kerf and dross but improves the surface roughness.

In order to reduce the amount of dross attached to the cutting edge, a mathematic model for the dross dimensions was formulated. The dross height and the dross diameter were analyzed and compared with the experimental results. Both dimensions increase with the increasing of the pulse peak power.

\section{Acknowledgements}

The authors would like to express their gratitude to the GREP research group from the UdG, the Tecnologico de Monterrey for the facilities provided during the experiments. This work was partially carried out with the grant supports from the European Commission project IREBID (FP7-PEOPLE-2009-IRSES-247476) and the Spanish Science and Innovation Minister project TECNIPLAD (DPI2009- 09852).

\section{References}

[1] G. Tawari, J.K. Sarin Sundar, G. Sundararajan, S.V. Joshi. Influence of process parameters during pulsed Nd:YAG laser cutting of nickel-base superalloys. Journal of Materials Processing Technology 170 (2005) 229-239.

[2] K.F. Kleine, B. Whitney, K.G. Watkins. Use of fiber lasers for micro cutting applications in medical device industry. 21st International Congress on Applications of Lasers and ElectroOptics, (2002).

[3] N. Muhammad, D. Whitehead, A. Boor, L. Li. Precision Machine Design. Comparison of dry and wet fiber laser profile cutting of thin $316 \mathrm{~L}$ stainless steel tubes for medical device applications. Journal of Materials Processing Technology 210 (2010) 2261-2267.

[4] H. Meng, J. Liao, Y. Zhou, Q. Zhang. Laser micro-processing of cardiovascular stent with fiber laser cutting system. Optics \& Laser Technology 41 (2009) 300-302.

[5] M. Baumeister, K. Dickman, T. Hoult. Fiber laser micro-cutting of stainless steel sheets. Journal of Applied Physics. A 85, 121-124 (2006).

[6] L.D. Scintilla, D. Sorgente, L. Tricarico. Experimental Investigation On Fiber Laser Cutting Of Ti6Al4V Thin Sheet. Journal of Advanced Materials Research Vols. 264-265 (2011) pp 12811286.

[7] J. Powell, S.O. Al-Mashikhi, K.T.Voisey. Fibre laser cutting of thin section mild steel: An explanation of the 'striation free' effect. Optics and Lasers in Engineering 49 (2011) 1069-1075 
[8] Y. Yan, L. Li, K. Sezer, D. Whitehead, L. Ji, Y. Bao, Y. Jiang. Experimental and theoretical investigation of fibre laser crack-free cutting of thick-section alumina. International Journal of Machine Tools \& Manufacture 51 (2011) 859-870

[9] Y.P. Kathuria. Laser microprocessing of metallic stent for medical therapy. Journal of Materials Processing Technology 170 (2005) 545-550.

[10] R. Pfeifer, D. Herzog, M. Hustedt, S. Barcikowski. Pulsed Nd:YAG laser cutting of NiTi shape memory alloys-Influence of process parameters. Journal of Materials Processing Technology 210 (2010) 1918-1925.

[11] Lv. Shanjin, W. Yang. An investigation of pulsed laser cutting of titanium alloy sheet. Optics and Lasers in Engineering 44 (2006) 1067-1077.

[12] K.C. Yung, H.H. Zhu, T.M. Yue. Theoretical and experimental study on the kerf profile of the laser micro-cutting NiTi shape memory alloy using $355 \mathrm{~nm}$ Nd:YAG. Smart Materials and Structures 14 (2005) 337-342.

[13] N. Muhammad, D. Whitehead, A. Boor, W. Oppenlander, Z. Liu, L. Li. Picosecond laser micromachining of nitinol and platinum-iridium alloy for coronary stent applications. Applied Physics A (2012) 106:607-617.

[14] C. Lia, S. Nikumbb, F. Wong. An optimal process of femtosecond laser cutting of NiTi shape memory alloy for fabrication of miniature devices. Optics and Lasers in Engineering 44 (2006) 1078-1087.

[15] H. Huang, H.Y. Zheng, G.C. Lim. Femtosecond laser machining characteristics of Nitinol. Applied Surface Science 228 (2004) 201-206.

[16] A. Raval, A. Choubey, C. Engineer, D. Kothwala. Development and assessment of 316LVM cardiovascular stents. Materials Science and Engineering A 386 (2004) 331-343.

[17] L.D. Scintilla, L. Tricarico. Experimental investigation on fiber and $\mathrm{CO} 2$ inert gas fusion cutting of AZ31 magnesium alloy sheets. Optics \& Laser Technology 46 (2013) 42-52.

[18] B.S. Yilbas, B.J. Abdul Aleem. Dross formation during laser cutting process. Journal of Physiscs D: Applied Physics 39 (2006) 1451-61.

[19] G. Tani, L. Tomesani, G. Campana, A. Fortunato. Quality factors assessed by analytical modelling in laser cutting. Thin Solid Films 453-454 (2004) 486-491.

[20] D. Schuöcker, J. Aichinger, R. Majer. Dynamic phenomena in laser cutting and process performance. Physics Procedia 39 (2012) 179-185.

[21] S.Z. Shuja, B.S. Yilbas, O. Momin. Laser heating of a moving slab: influence of laser intensity parameter and scanning speed on temperature field and melt size. Optics \& Laser Technology 49 (2011) 265-272.

[22] M. Shikida, T. Yokota, J. Naito, K. Sato. Fabrication of a stent-type thermal flow sensor for measuring nasal respiration. Journal of Micromechanics and Microengineering 20 (2010) 055029. 
[23] D. Teixidor, F. Orozco, T. Thepsonthi, J. Ciurana, C.A. Rodriguez, T. Özel. Effect of process parameters in nanosecond pulsed laser micromachining of PMMA-based microchannels at near-infrared and ultraviolet wavelengths. International Journal of Advanced Manufacturing Technology (2012) 170-012-4598-x 